\title{
Research article \\ Comparison of serum calcium, magnesium level and total antioxidant activity in pregnant women with and without gestational diabetes mellitus
}

\author{
Deepa K. Kamath ${ }^{1}$, Muralidhar V. Pai ${ }^{2}$, Saleena Ummer ${ }^{3}$, Saritha Kamath U. ${ }^{4}$ \\ ${ }^{1}$ Post graduate, ${ }^{3}$ Professor, ${ }^{4}$ Associate Professor, Medical Laboratory Technology, Manipal College of Health Professions, \\ Manipal Academy of Higher Education (MAHE), Manipal, 576 104, Karnataka, India \\ ${ }^{2}$ Professor, Department of Obstetrics and Gynaecology, Kasturba Medical College, Manipal, Manipal Academy of Higher \\ Education (MAHE), Manipal, Karnataka, India
}

(Received: May $2021 \quad$ Revised: June $2021 \quad$ Accepted: June 2021)

Corresponding author: Saritha Kamath U. Email: saritha.kamath@manipal.edu

\begin{abstract}
Introduction and Aim: Pregnancy is a normal physiological state during which women experience a lot of internal bodily changes. Gestational diabetes mellitus (GDM) is a complication of pregnancy which is characterized by hyperglycemia due to an error in carbohydrate metabolism. During pregnancy changes in metabolism may induce oxidant stress and it may increase in GDM. The present study was done to compare serum calcium, magnesium and total antioxidant activity in pregnant women with and without hyperglycemia.
\end{abstract}

Materials and Methods: The study was conducted for a period of one year. Total 67 participants were included and their serum samples were analyzed for serum calcium magnesium and total antioxidant activity. Among these 29 were GDM (43\%) and 38 were non-GDM (57\%). All study subjects were on supplementation of calcium, iron and folic acid.

Results: In our study, there were no significant difference in serum calcium, magnesium and antioxidant activity between GDM and non GDM women.

Conclusion: Calcium, magnesium and antioxidant activity were maintained well both in pregnant women GDM and non GDM, this may be due to beneficial effect of calcium, iron, folic acid supplementation.

Keywords: Gestational diabetes; delivery; pregnancy; hyperglycemia.

\section{INTRODUCTION}

$\mathrm{P}$ regnancy is a normal physiological state during this period women experience a lot of bodily changes. This is a period of rapid growth through cell maturation, differentiation both in mother and growing fetus (1), (2). Hyperglycemia occurs the first time during pregnancy is also known as GDM. It is associated with the risk of complications during pregnancy, delivery and adverse perinatal outcome. It can also cause intrauterine growth retardation, preterm delivery, congenital anomalies, and delay in organ developments and reduced immunity (3), (4). The requirement of micronutrient is more than the normal during pregnancy if there is a deficiency it can lead to several adverse effects both on mother and fetus. It may limit weight gain, adverse effect on fetal growth, neural tube defect, anemia, increase in blood pressure, and complications at delivery, or even death in mother (5). Minerals play an important role in the body especially in bone construction, regulation of blood sugar, transport of oxygen, regulation of chemical reaction, protection of cells from oxidative damage and regulation of immune system function. (6).

Micronutrient deficiency during pregnancy leads to some increased risks which include preeclampsia, fetal growth restriction, anemia, pregnancy-induced hypertension, increased labor complications, and maternal and fetal mortality (7). Calcium requirement is more during pregnancy for the proper skeletal mineralization and growth in the fetus. To meet this demand there will be intestinal absorption nearly double than the normal during pregnancy. $(8,9)$ The second major intracellular cation is magnesium. It plays a vital role in carbohydrate metabolism and blood pressure regulation. During pregnancy, it is also having a beneficial role in the relaxation of muscles, vasodilatation and decreased vascular resistance. Deficiency of magnesium during pregnancy can lead to complications such as preeclampsia, preterm delivery, gestational diabetes etc. $(10,11)$ Oxidative stress is an imbalance between total antioxidant capacity and pro-oxidants, it is having an important impact on pregnancy from the initial stage till labor and delivery (12).

Damage to the antioxidant system occurs in gestational diabetics may be due to the increase of free radicals because of increased lipid peroxidation. (13). In this study, we tried to compare the serum calcium, magnesium and total antioxidant levels in pregnant women with and without GDM.

\section{MATERIALS AND METHODS}

This study was conducted for a period of one year after getting approval from the institutional research committee and institutional ethical committee and clinical trial registration (CTRI/2018/07/014893). 
Pregnant women with abnormal glucose challenge test or glucose tolerance test were included as gestational diabetes mellitus. Pregnant women with normal fasting/postprandial blood glucose/glucose challenge test were considered as non-gestational diabetes mellitus. The study population included pregnant women with and without GDM attending OBG department during $2^{\text {nd }}$ or $3^{\text {rd }}$ trimester (routine checkup) with blood requisition forms for routine blood investigations. We have obtained informed consent from the pregnant who were having a requisition for plain sample, we asked their Informed consent to use their left-over serum sample from the laboratory (after completing the required investigations). Serum samples were collected from the laboratory after getting permission from the laboratory in charge. Collected serum samples were stored in a deep freezer at $-20^{\circ} \mathrm{C}$ till the analysis and used for estimation of calcium, magnesium and total antioxidant activity.
Estimation of serum calcium, magnesium level was done by using Kit method (Agappe Diagnostics Company) and Total antioxidant activity by manual method (14) Clinical and other laboratory data were obtained from the medical records as per the proforma after obtaining permission from Chief Operating Officer, Kasturba Hospital, Manipal, Manipal Academy of Higher Education.

\section{RESULTS}

The study conducted during the period of one year where pregnant women with and without hyperglycemia attending OBG department, Kasturba Hospital Manipal, Manipal Academy of Higher Education, Manipal were included. A total of 67 participants were included and their serum samples were analyzed for Calcium, Magnesium and Total antioxidant activity. Among these 29 were GDM $(43 \%)$ and 38 were non-GDM (57\%).

Table 1: Baseline data of participants in GDM and Non GDM groups

\begin{tabular}{|c|c|c|}
\hline Particulars & GDM (Cases) & Non GDM (Controls) \\
\hline Age & & \\
\hline $20-30$ year & $62 \%$ & $66 \%$ \\
$30-40$ year & $38 \%$ & $34 \%$ \\
\hline BMI & 24.97 & 24.94 \\
\hline Primi gravid & & \\
\hline $2^{\text {nd }}$ trimester & $31 \%$ & $26 \%$ \\
$3^{\text {rd }}$ trimester & $69 \%$ & $74 \%$ \\
\hline Medications (Calcium, folate and iron) & $97 \%$ & $97 \%$ \\
\hline
\end{tabular}

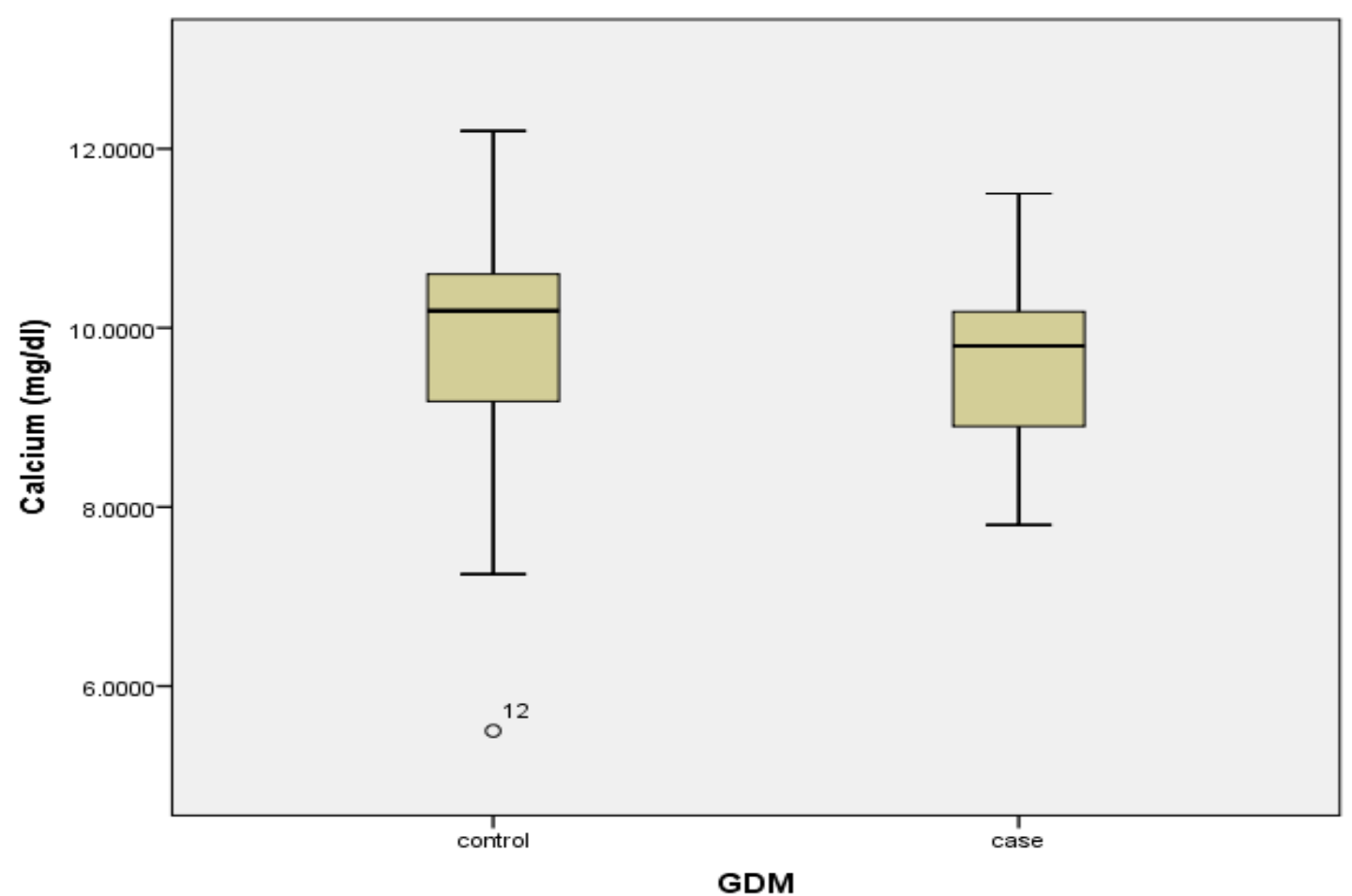

Fig. 1: Calcium (mg/dl) levels in GDM (cases) and non GDM (control) groups 


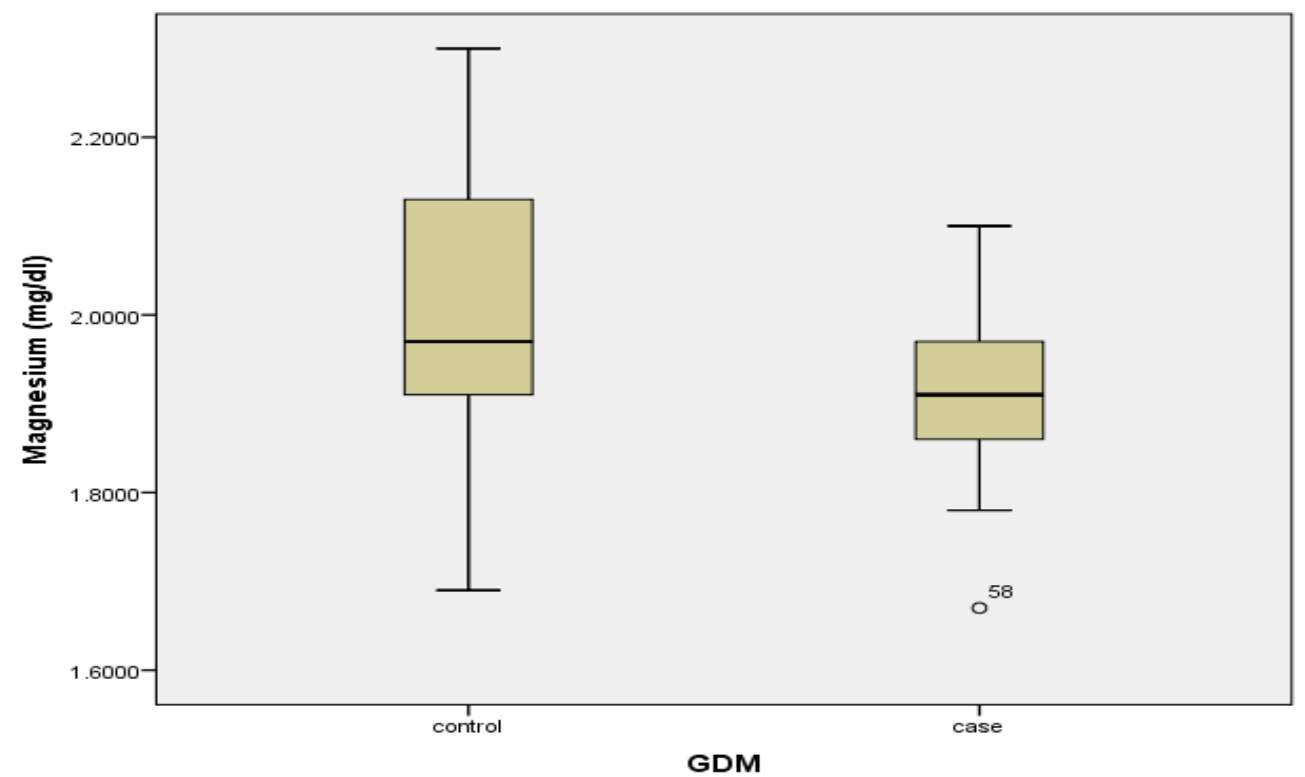

Fig. 2: Magnesium (mg/dl) levels in GDM (cases) and non GDM (control) groups

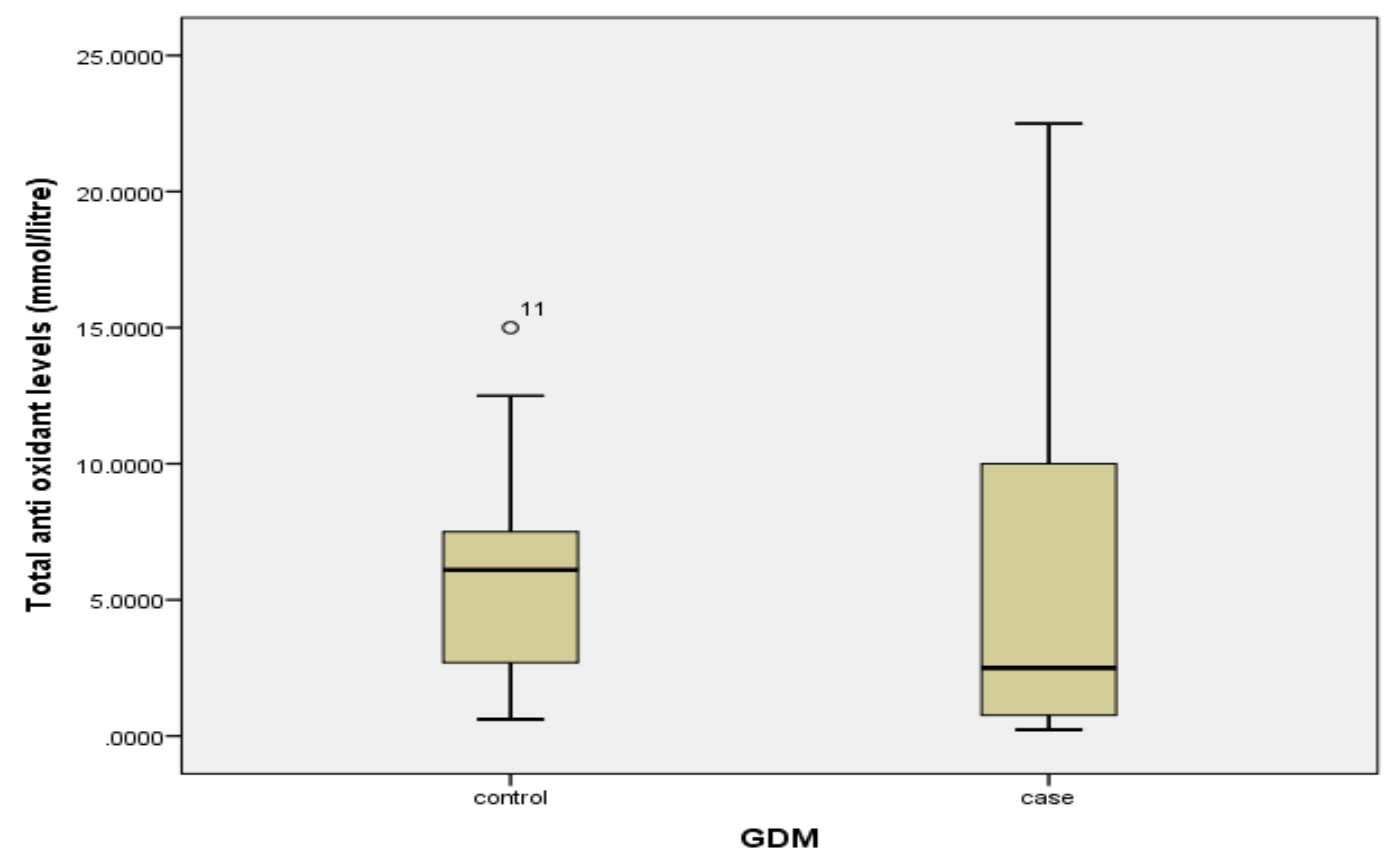

Fig. 3: Total antioxidant activity (mmol/L) levels in GDM (cases) and non GDM (control) groups

Table 2: Comparison of serum calcium, magnesium and antioxidant level between GDM and non GDM

\begin{tabular}{|c|c|c|c|}
\hline & $\begin{array}{c}\text { GDM (Cases) } \\
\text { Mean } \pm \text { SD }\end{array}$ & $\begin{array}{c}\text { Non GDM (Controls) } \\
\text { Mean } \pm \text { SD }\end{array}$ & p value \\
\hline Calcium $(\mathrm{mg} / \mathrm{dl})$ & $9.61 \pm 1.92$ & $9.84 \pm 2.66$ & 0.171 \\
\hline Magnesium $(\mathrm{mg} / \mathrm{dl})$ & $1.90 \pm 0.14$ & $1.99 \pm 0.36$ & $0.050^{*}$ \\
\hline Total antioxidant activity(mmol/litre) & $6.82 \pm 6.58$ & $5.74 \pm 5.60$ & 0.372 \\
\hline
\end{tabular}

* Significant as $\mathrm{p}$ value is $<0.05$

The data were analyzed related to serum calcium, magnesium and antioxidant activity using KS (Kolmogorov-Smirnov) to check for the normality between GDM and non-GDM group. It showed that, there is no difference for between GDM and nonGDM group for serum calcium, magnesium and total antioxidant activity. (Fig. 1 to Fig.3) Using Mann-Whitney $U$ test serum calcium, magnesium and total antioxidant activity were compared in GDM and Non-GDM groups showed statistically significant only for serum magnesium as in table 2 .

\section{DISCUSSION}

Pregnancy is a period where a lot of changes occurs in women's physiology. It is also a period of increased metabolic needs of micronutrients for the growth of the fetus. The study showed that the level of minerals and micronutrients alters during pregnancy. Deficiency of these trace elements, minerals and 
inadequate diet can have an impact on the mother and fetus. It includes hypertension, complications of labor, anemia or even death. Furthermore, it can lead to other consequences such as preterm delivery, growth retardation, congenital anomalies, reduced immunity, and abnormal organ developments (1-4).

For the normal functioning of the human body, calcium and magnesium are essential minerals. Calcium is also required for muscle contraction and maintaining water balance. For many enzyme systems functioning, magnesium is the essential co-factor. It's also required for neurochemical transmission and vasodilation. (15) Alteration in the level of estragon and progesterone hormones during pregnancy may alter the level of calcium and it may affect mother and fetus (16).

In the present study it was found that the calcium level in serum was within the normal limit in both pregnant women with GDM and non GDM. This was in accordance with the study conducted by Tabrizi et al., (17). This may be because of supplementation of calcium during pregnancy in our studied group (Table.2). However, we have observed a change in calcium level during the second and third trimester. This was in accordance with the study conducted by Liu et al., (18).

The study conducted at Iran observed that $13 \%$ of pregnant women had decreased calcium level. (17) Another study found that $19 \%$ of pregnant women were with hypocalcemia. Factors affected the low calcium levels in their study was the deficiency of vitamin $\mathrm{D}$, inadequate calcium intake through diet or things that interfere with calcium absorption (19). The reason for this may be due to their diet rich in wheat, staple cereal. It can impair the absorption of calcium from the intestine. It may be also because of the increased phytic acid level in wheat. Healthy and balanced diet is crucial to maintain nutrient level during pregnancy (20). Our study result is different from this study which may be because of supplementation of both calcium and vitamin D from the beginning of pregnancy. Our study is in accordance with the study conducted in India by Siddiqqi et al., which showed that serum calcium level was within the normal limit both in GDM and NonGDM groups with no statistically significant difference

Our study found that the magnesium level was within the normal limit in both pregnant women with GDM and non GDM groups. This was in accordance with the study by Tabrizi et al., (17). However, we have observed a change in serum magnesium level during the second and third trimester. This was in accordance with the study conducted by Liu et al., (18). Study by Nabouli et al also concluded that development of GDM cannot be explained by low total serum magnesium. (22).

Another study conducted at Korea by Yang et al., showed that decreased magnesium is often seen in Type 2 diabetes mellitus (23). Our study observed that the magnesium level was within the normal limit in both GDM and non GDM groups (Table.2).

Karacay et al., showed that total antioxidant status was decreased in GDM compared to normal pregnancies (24). Parast and Paknahad compared antioxidant capacity among GDM and non GDM and they observed that TAC level in women with GDM was significantly lower than non-GDM (25). Our study found that total antioxidant activity level was within the normal limit in both GDM and non GDM groups (Table 2). This is also again may be due to the awareness and proper care taken during pregnancy period.

\section{CONCLUSION}

Calcium, iron and folic acid supplementation in women had beneficial effects on their metabolic profile. In our study, there was no significant difference in serum calcium, magnesium and antioxidant activity between GDM and non-GDM women. All were on proper supplementation of micronutrients. This indicates that they may be having sufficient knowledge about proper nutrition during pregnancy. Most of the tertiary care hospitals are prescribing vitamins and minerals supplementation during pregnancy. Our study was done in a tertiary care center and hence there is a need for conducting a study among rural and urban area to know more about their knowledge.

\section{ACKNOWLEDGMENT}

Authors are thankful to the participants who had given consent and participated in this study.

\section{CONFLICT OF INTEREST}

The authors declare no conflicts of interest.

\section{REFERENCES}

1. Soma Pillay, P., Nelson-Piercy, C., Tolppanen, H., Mebazaa, A. Physiological changes in pregnancy. Cardiovasc J Afr. 2016; 27(2): 89-94

2. Moya, J., Phillips, L., Sanford, J., Wooton, M., Gregg, A., Schuda, L. A review of physiological and behavioral changes during pregnancy and lactation: Potential exposure factors and data gaps. J Expo Sci Environ Epidemiol. 2014; 24(5): 449-458.

3. Farrar, D. Hyperglycemia in pregnancy: prevalence, impact and management challenges. Int J Women's Health. 2016; 8: 519-527.

4. HAPO Study Cooperative Research Group, "Hyperglycemia and Adverse Pregnancy Outcome (HAPO) study: associations with neonatal anthropometrics," Diabetes. 2009; 58: 453-459.

5. Mousa, A, Naqash, A. Macronutrient and Micronutrient Intake during Pregnancy: An Overview of Recent Evidence. Nutrients. 2019; 11(443): 1-20.

6. Siddiqui, K., Bawazeer, N., Joy, S. S. Variation in Macro and Trace Elements in Progression of Type 2 Diabetes. Sci World J. 2014; 1-9.

7. King, J. C. Determinants of maternal zinc status during pregnancy-. The American Journal of Clinical Nutrition. 2000 May 1; 71(5): 1334S-1343S. 
8. Kovacs, C. S. Maternal mineral and bone metabolism during pregnancy, lactation, and post-weaning recovery. Physiological reviews. 2016 Feb 17; 96 (2): 449-547.

9. Hacker, A. N., Fung, E. B., King, J. C. Role of calcium during pregnancy: maternal and fetal needs. Nutrition reviews. 2012 Jul 1; 70(7): 397-409.

10. Swaminathan, R. Magnesium metabolism and its disorders. Clin Biochem Rev. 2003; 24(May): 47-66.

11. Dalton, L. M., Deirdre, M. N., Gaydadzhieva, G. T., Mazurkiewicz, O. M., Leeson, H., Wright, C. P. Magnesium in pregnancy. Nutr Rev. 2016; 74(9): 549557.

12. Duhig, K., Chappell, L. C., Shennan, A. H. Oxidative stress in pregnancy and reproduction. Obstet Med. 2016; 9(3): 113116.

13. Raijmakers, M. T., Peters, W. H., Steegers, E. A., Poston, L. Amino thiols, detoxification and oxidative stress in preeclampsia and other disorders of pregnancy. Current pharmaceutical design. 2005 Mar 1; 11(6): 711-734.

14. Koracevic, D., Koracevic, G., Djordjevic, V., Andrejevic, S., Cosic, V. Method for the measurement of antioxidant activity in human fluids Clin Pathol 2001; 54: 356-361

15. Black, R. E. Micronutrients in pregnancy. Br J Nutr 2001 85(12):S193-S197.

16. WHO (1996) Trace elements in human nutrition and health. WHO, Geneva, Switzerland.

17. Tabrizi, F. M., Pakdel, F. G. Serum level of some minerals during three trimesters of pregnancy in Iranian women and their new borns: a longitudinal study. Indian Journal of Clinical Biochemistry. 2014 Apr 1; 29(2): 174-180.

18. Liu, J., Yang, H., Shi, H., Shen, C., Zhou, W., Dai, Q., et al., Blood copper, zinc, calcium, and magnesium levels during different duration of pregnancy in Chinese. Biological trace element research. 2010 Jun 1; 135(1-3): 31-37.

19. Ainy, E., Ghazi, A. A. M., Azizi, F., Changes in calcium, 25 $(\mathrm{OH})$ vitamin D3 and other biochemical factors during pregnancy. J Endocrinol Invest. 2006; 29(4): 303-307.

20. Al, Hasan, S. M., Hassan, M., Saha, S., Islam, M., Billah, M., Islam, S. Dietary phytate intake inhibits the bioavailability of iron and calcium in the diets of pregnant women in rural Bangladesh: A cross-sectional study. BMC Nutr 2016; 2(1): $1-10$.

21. Siddiqi, S. S., Borse, A. G., Pervez, A., Anjum, S. A study of bone turnover markers in gestational diabetes mellitus. Indian journal of endocrinology and metabolism. 2017 Jan; 21(1): 38.

22. Nabouli, M. R., Lassoued, L., Bakri, Z., Moghannem, M. Modification of total magnesium level in pregnant Saudi women developing gestational diabetes mellitus. Diabetes \& Metabolic Syndrome: Clinical Research \& Reviews. 2016 Oct 1; 10(4): 183-185.

23. Yang, S. J., Hwang, S. Y., Baik, S. H., Lee, K. W., Nam, M. S., Park, Y. S., et al., Serum magnesium level is associated with type 2 diabetes in women with a history of gestational diabetes mellitus: the Korea National Diabetes Program study. Journal of Korean Medical Science. 2014 Jan 1; 29(1): 84-89.

24. Karacay, O., Dincel, A. S., Karcaaltincaba, D., Sahin, D., Yalvaç, S., Akyol, M., et al. A quantitative evaluation of total antioxidant status and oxidative stress markers in preeclampsia and gestational diabetic patients in 24-36 weeks of gestation. Diabetes Res Clin Pract. 2010 Sep; 89(3): 231238.

25. Parast, V. M., Paknahad, Z. Antioxidant status and risk of gestational diabetes mellitus: a case-control study. Clinical nutrition research. 2017 Apr 1; 6(2): 81-88. 